Article

\title{
Self-Assembly of Gold Nanocrystals into Discrete Coupled Plasmonic Structures
}

\author{
Carola Schopf, Ethel Noonan, Aidan J. Quinn and Daniela Iacopino * \\ Tyndall National Institute, University College Cork, Cork, Ireland; caro.schopf@gmail.com (C.S.); \\ etsnoonan2@gmail.com (E.N.); aidan.quinn@tyndall.ie (A.J.Q.) \\ * Correspondence: daniela.iacopino@tyndall.ie; Tel.: +353-(0)21-2346182
}

Academic Editors: Roberto Comparelli, Lucia Curri and Marinella Striccoli

Received: 25 July 2016; Accepted: 31 August 2016; Published: 14 September 2016

\begin{abstract}
Development of methodologies for the controlled chemical assembly of nanoparticles into plasmonic molecules of predictable spatial geometry is vital in order to harness novel properties arising from the combination of the individual components constituting the resulting superstructures. This paper presents a route for fabrication of gold plasmonic structures of controlled stoichiometry obtained by the use of a di-rhenium thio-isocyanide complex as linker molecule for gold nanocrystals. Correlated scanning electron microscopy (SEM) — dark-field spectroscopy was used to characterize obtained discrete monomer, dimer and trimer plasmonic molecules. Polarization-dependent scattering spectra of dimer structures showed highly polarized scattering response, due to their highly asymmetric $\mathrm{D}_{\infty \mathrm{h}}$ geometry. In contrast, some trimer structures displayed symmetric geometry $\left(D_{3 h}\right)$, which showed small polarization dependent response. Theoretical calculations were used to further understand and attribute the origin of plasmonic bands arising during linker-induced formation of plasmonic molecules. Theoretical data matched well with experimentally calculated data. These results confirm that obtained gold superstructures possess properties which are a combination of the properties arising from single components and can, therefore, be classified as plasmonic molecules.
\end{abstract}

Keywords: plasmonics; gold nanocrystals; self-assembly; plasmonic molecules; coupled structures

\section{Introduction}

The field of plasmonics has grown in the last two decades, driven by the prospect of controlling the properties of light with nanometre-scale precision by coupling electromagnetic fields to the oscillation of free electrons in metals. This has led to gain fundamental understanding into the interaction between light and matter at the nanoscale, allowed the diffraction limit to be beaten, produced ways to modify the properties of light emitters and enabled materials with optical properties with no counterpart in nature to be developed [1,2].

Single metal nanoparticles are the smallest unit to support plasmon-based interactions. Isolated or randomly aggregated metal nanoparticles display peculiar opto-electronic properties useful for numerous applications. However, the real interest in the last decade has concentrated on development of methodologies for the controlled chemical assembly of plasmonic nanoparticles into well-defined superstructures with predictable spatial geometry. Slow and controlled solvent evaporation of surfactant stabilized metal nanoparticle solutions has been used by our group and others to fabricate ordered large two- and three-dimensional superstructures with controlled geometries [3-5]. Another approach for fabrication of plasmonic structures of controlled dimension and geometry is the bottom-up assembly of chemically synthesized metal nanocrystals into higher-order structures $[6,7]$. The interest for this approach relies on the numerous applications of resulting structures ranging from surface enhanced spectroscopies [8-10], optical circuitry [11], biological sensors [12,13] and 
metamaterials [14,15]. In addition, new and complex self-assembled materials may exhibit novel properties arising from the combination of the individual component properties. In fact, these structures can be treated as plasmonic molecules, where the combination of localized surface plasmon resonances (LSPR) of individual nanostructures interact to form new coupled excitations, similar to the way electrons interact in molecules to form bonds [16]. The most common approach for self-assembly of metal nanoparticles exploits the known affinity between gold and sulfur groups thus relying on the use of thiol-functionalized linking molecules. Numerous molecules containing thiol and dithiol groups were used as linkers including aliphatic [17] and aromatic thiols [18], biological compounds [19,20] and single strand oligonucleotides [21,22]. The addition of controlled aliquots of such linkers to nanocrystal solutions gave rise to controlled growth and formation of geometrically well-defined nanocrystal clusters. Isothiocyanide $(-\mathrm{N}=\mathrm{C}=\mathrm{S}$ ) groups are also known to possess high affinity versus gold. So far this affinity has mostly been exploited to attach organic chromophores to colloidal gold nanocrystals or to nanoscale electrodes for surface-enhanced Raman scattering (SERS) applications [23], formation of metal junctions for molecular electronic applications [24], bioimmunoassays [25] and cancer therapy applications [26]. However, recent theoretical work by Sikora et al. showed that bis-isothiocyanide molecules can also be used as alternative to dithiols as conjugation molecules for bridging two or more nanocrystals together [27], whereby the lowest reactivity of isothiocyanide groups compared to thiols for citrate stabilized gold nanoparticles was shown useful to achieve good control in the growth rate of nanoparticle clusters.

In this paper we have used a bis-isocyanide Rhenium complex to synthesize gold nanocrystal clusters of defined stochiometry and geometry, also known as plasmonic molecules. Increasing aliquots of linker molecule were mixed with nanocrystal solutions and the evolution of cluster formation was monitored by UV-vis spectroscopy. Correlated scanning electron microscopy (SEM)—dark-field spectroscopy was used to characterize discrete monomer, dimer and trimer plasmonic molecules. Compared to the scattering spectra of single nanocrystals, additional features appeared in the assembled structures, due to plasmonic coupling between nanocrystals. Polarization-dependent scattering spectra of dimer structures showed highly polarized scattering response, due to their highly asymmetric geometry $\left(\mathrm{D}_{\infty \mathrm{h}}\right)$. In contrast, trimer structures displayed symmetric geometry $\left(\mathrm{D}_{3 \mathrm{~h}}\right)$, which showed small polarization-dependent response. The experimental data from both dimer and trimers matched well with theoretically calculated data, which were used to further understand and attribute the origin of plasmonic bands arising during linker-induced formation of plasmonic molecules.

\section{Results}

In assembled nanostructures the geometry and monodispersity of the nanocrystal building blocks is critical to control the self-assembly process and for accurate comparison between experimental and theoretical data. Used gold nanocrystals had an average size of $60 \mathrm{~nm}$ and a maximum absorption band centered at $540 \mathrm{~nm}$ (see Figure S1). Therefore, prior to self-assembly experiments statistical correlated scanning electron microscopy (SEM) and darkfield microscopy were used to assess the size and shape distribution of nanocrystals in the used solution (see Figures S2 and S3). The majority of the particles ( $88 \%$ occurrence) were spherical or near-spherical, with a measured plasmon resonance peak wavelength between $526 \mathrm{~nm}$ and $536 \mathrm{~nm}$. Hexagons (9\% occurrence) displayed scattering peaks in the range $554-572 \mathrm{~nm}$ and triangles (2\% occurrence) in the range $583-606 \mathrm{~nm}$. Small percentage of tetrahedrons (1\%) were also found exhibiting the same resonance wavelength $(534 \mathrm{~nm})$ as spherical particles with, however, a slightly broader full width half maximum (FWHM). 
Scheme 1 shows a graphical representation of the gold nanocrystals and the $\operatorname{Re}_{2}(\mathrm{DMAA})_{4}(\mathrm{NCS})_{2}$ linker molecule used in this work as well as a schematic of the coupled structures formed during the assembly process comprising one-nanocrystal (monomer), two nanocrystals (dimers), three nanocrystals (trimers) and higher order $n$-mer structures. The attachment of the linker molecule to the gold nanocrystals was promoted by the high affinity of the sulphur atom of the isothiocyanate group for gold. The distance between the end thiol groups was calculated from X-ray crystallography data to be $1.2 \mathrm{~nm}[28]$.

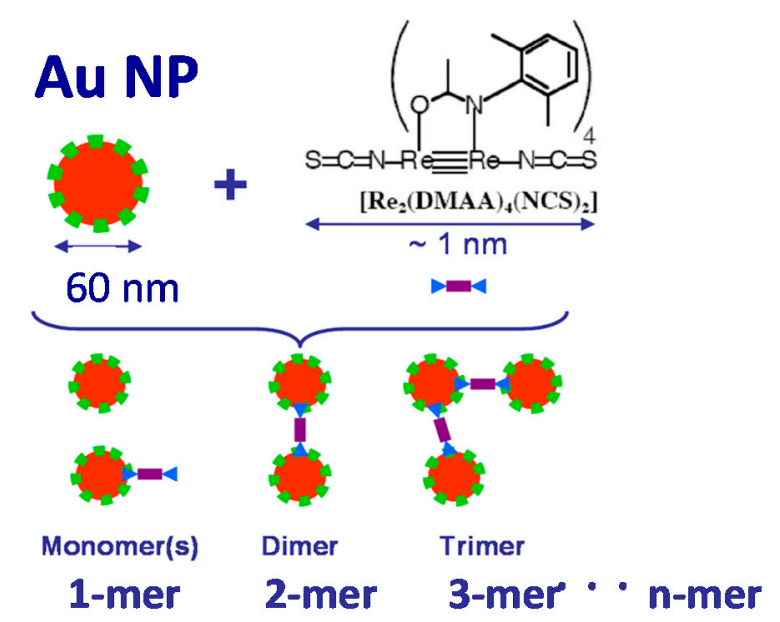

Scheme 1. Molecular structure of $\operatorname{Re}_{2}(\mathrm{DMAA})_{4}(\mathrm{NCS})_{2}$ and schematic of n-mers formation.

Figure 1a shows the formation of gold nanocrystal structures monitored by UV-vis spectroscopy. The spectrum of isolated nanocrystals in water solution ( $60 \mathrm{~nm}$ diameter) was characterized by a plasmon resonance peak at $540 \mathrm{~nm}$. Progressive addition of linker molecule resulted in formation of an additional band at $675 \mathrm{~nm}$, representative of the coupling between nanocrystals and formation of nanocrystal clusters. The near-infrared band reached its maximum after addition of $40 \mu \mathrm{mol}$ of linker molecule, corresponding to a linker molecule/nanocrystal ratio of 6179:1. Theoretical calculations performed on smaller nanocrystals showed that the maximum coverage of linker molecule for $40 \mathrm{~nm}$ diameter nanocrystals was equal to 4273 , which could be in agreement with the experimentally added amount of 6179 for a $60 \mathrm{~nm}$ diameter particle [27]. This value corresponded to a nanocrystal coverage of $4.9 \%$ calculated approximating the nanocrystal to a sphere of radius $30 \mathrm{~nm}$ and the linker molecule to a sphere of $0.6 \mathrm{~nm}$ radius and assuming that all added linker molecule was bound to the nanocrystal surface. The range of cluster size and shapes obtained was analyzed by SEM. As shown in the inset of Figure 1a, a number of structures formed, including dimers, trimers, quadrumers and small clusters. Formed n-mer structures are also shown in a representative image in Figure $1 \mathrm{~b}$ and in the additional images reported in Figure S4: Trimers were usually arranged in triangular shape, some of them showing high $D_{3 h}$ symmetry, and quadrumers usually arranged in $D_{\infty \mathrm{h}}$ symmetry, although more geometrical arrangements would be possible for such structures. Statistical analysis reported in Figure 1c, performed on 100 structures, showed that the majority of structures in solution were single nanocrystals $(70 \%)$, which explains the small change of the plasmon band at $540 \mathrm{~nm}$ upon addition of linker molecule. Dimers constituted ca. $20 \%$ of the formed structures, whereas the remaining $10 \%$ was constituted by trimers, quadrumers and larger clusters. Control experiments on unmodified nanocrystals prepared without addition of the linker molecules yielded over $95 \%$ of monomers with a low incidence of dimers and negligible incidence of higher order $n$-mers (data not shown). 

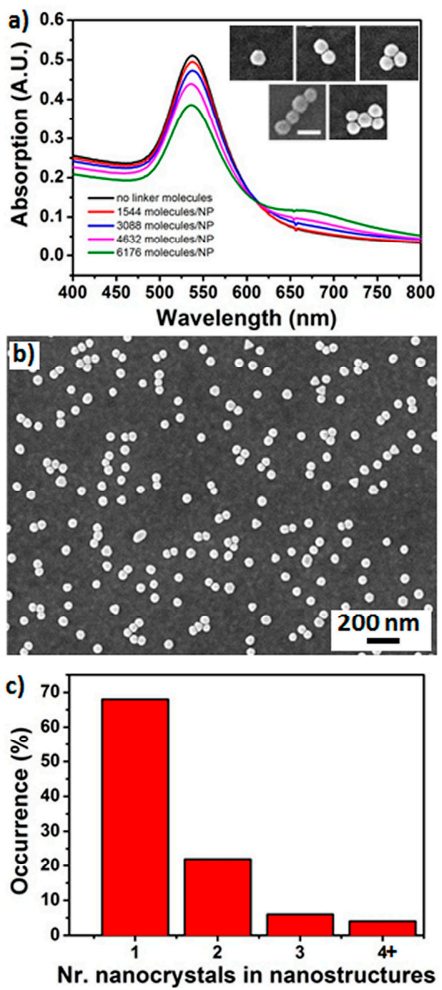

Figure 1. (a) UV-vis spectra of gold nanoparticles with increasing amount of added Rhenium ligand showing progressive formation of n-mers. Inset: Representative SEM images of formed n-mers (scale bar: $100 \mathrm{~nm}$ ); (b) SEM image of formed n-mers deposited on $\mathrm{SiO}_{2}$ substrate; (c) statistical analysis of formed gold nanocrystal structures.

Bringing nanocrystals in close proximity of each other, within 2.5 times the particle diameter to each other, led to coupling of their plasmon modes and resulted in more complex optical signatures. In order to analyse such features at discrete level correlated darkfield spectroscopy and SEM imaging of formed plasmonic molecules was performed. Representative scattering experimental and calculated spectra of a monomer, dimer and trimer formed by $60 \mathrm{~nm}$ gold nanocrystals are shown in Figure 2 the peak positions are noted in Table 1 . For all three structures the scattering curve was calculated using a boundary element method to solve Maxwell's equations [29]. The nanocrystals were discretized by spheres with 1444 vertices, a diameter of $60 \mathrm{~nm}$ and an inter-particle separation of $1 \mathrm{~nm}$, based on the linker molecule length. The dielectric function for the gold nanoparticles was taken from Johnson and Christy, the average dielectric constant of the environment was assumed as 1.33 [30].
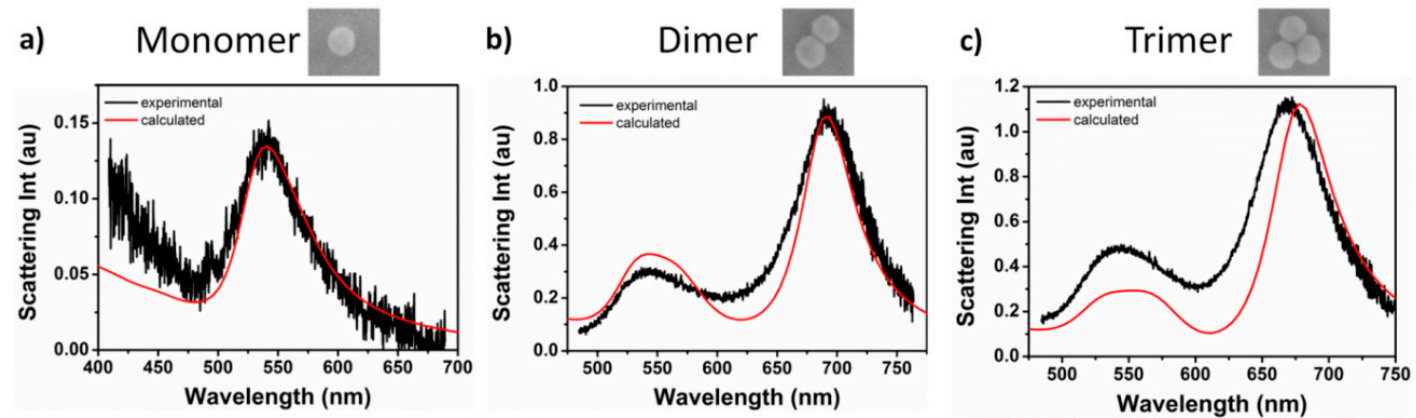

Figure 2. Scattering spectra of (a) a monomer; (b) a dimer; (c) a trimer gold nanocrystal structures. Experimental data (black) and calculated (red) spectra. Top row: Correlated SEM images of the corresponding structures. 
Table 1. Experimentally determined and calculated resonance wavelengths for gold nanocrystal monomer, dimer and trimer structures.

\begin{tabular}{lccc}
\hline & Monomer & Dimer & Trimer \\
\hline$\lambda_{\max , 1}(\exp$, calc) $(\mathrm{nm})$ & 540,541 & 541,544 & 542,551 \\
$\lambda_{\max , 2}(\exp$, calc) $(\mathrm{nm})$ &,-- & 693,691 & 669,678 \\
\hline
\end{tabular}

The experimental scattering spectrum of a representative nanocrystal monomer (Figure 2a, black curve) showed one peak at a resonance wavelength of $540 \mathrm{~nm}$. This value correlated well with the calculated resonance wavelength of $60 \mathrm{~nm}$ spherical particles, centered at $541 \mathrm{~nm}$ (Figure 2a, red curve). Two scattering peaks were experimentally observed in dimer structures Figure $2 \mathrm{~b}$ centered at $541 \mathrm{~nm}$ and $693 \mathrm{~nm}$, respectively. The correlated SEM image showed that the measured dimer structure was constituted by spherical particles of very close diameter size. For this reason the experimental peak wavelength positions correlated well with the calculated peak positions of $544 \mathrm{~nm}$ and $691 \mathrm{~nm}$. The experimental spectrum of a representative trimer of $D_{3 \mathrm{~h}}$ symmetry displayed two peaks centered at $542 \mathrm{~nm}$ and $551 \mathrm{~nm}$. Compared to the calculated values of $669 \mathrm{~nm}$ and $678 \mathrm{~nm}$, experimental data were red-shifted by $9 \mathrm{~nm}$. This could be related to small deviation from perfect equilateral triangle geometry or some breaking of the symmetry caused by slightly different size nanocrystals constituting the cluster.

In order to better analyze the origin of peak formation, scattering spectra of nanocrystal clusters were taken at different polarization angles, as shown in Figure 3. For the dimer structure Figure 3a the peak at $541 \mathrm{~nm}$ showed a small change in intensity with the change of the polariser angle, equivalent to a factor 1.7 difference in intensity between scattering maxima at crossed polarized positions. In contrast, the peak at $693 \mathrm{~nm}$ showed a dramatic change in intensity with the rotation of the polarizer angle. The peak intensity decreased as the incident light polarization was rotated from parallel to perpendicular to the long axis of the dimer. Specifically, the alignment of the polarization angle parallel to the long dimer axis resulted in the maximum intensity of the peak at $693 \mathrm{~nm}$ whose intensity was 25 times higher than the intensity displayed for an alignment of the polarization angle perpendicular to the long dimer axis. This suggested that the resonance wavelength at $693 \mathrm{~nm}$ generated as a result of the formation of a strongly coupled plasmon mode along the longitudinal axis of the dimer. Figure $3 \mathrm{c}$ shows a SEM image of the analyzed dimer with an overlaid plot of the plasmon peak intensity variation in dependence of the polarization angle. The plot clearly showed the generation of a strong dipole coupling parallel to the dimer long axis. In contrast, the lack of polarization dependence of the $540 \mathrm{~nm}$ plasmon mode was attributed to a weak dipole coupling perpendicular to the dimer long axis.
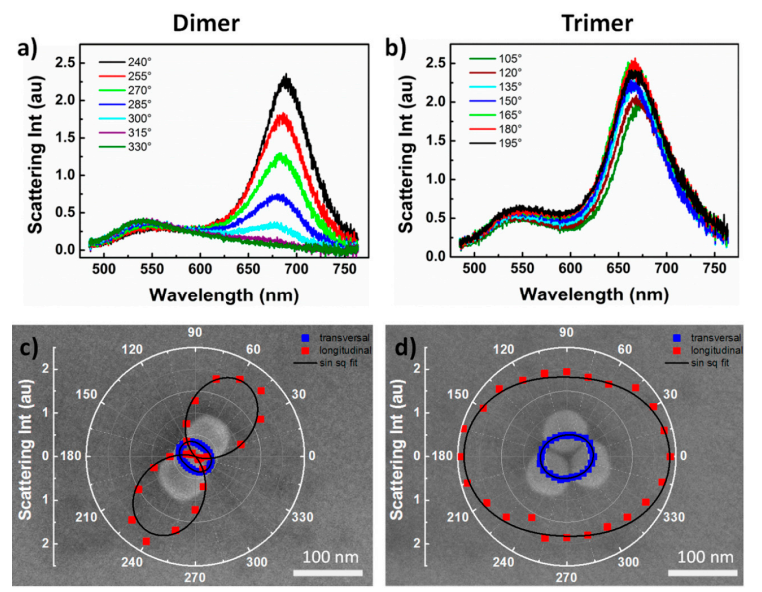

Figure 3. (a) and (b) polarization resolved scattering spectra of a $60 \mathrm{~nm}$ gold nanoparticle dimer and trimer. (c) and (d) SEM images of the analyzed nanoparticle dimer and trimer overlaid with the transversal and longitudinal peak plasmon intensity in dependence of the polarizer angle. 
The polarization dependent spectra of the $\mathrm{D}_{3 \mathrm{~h}}$ symmetry trimer (Figure $3 \mathrm{~b}$ ) showed a very small intensity change with the variation of the polarizer angle for both $542 \mathrm{~nm}$ and $669 \mathrm{~nm}$ peaks. This was expected, due to the increased anisotropy of $\mathrm{D}_{3 \mathrm{~h}}$ trimer structures compared to $\mathrm{D}_{\infty \mathrm{h}}$ dimer structures. Specifically, a perfectly symmetrical trimer would be expected to give rise to overlapping spectra in all polarization directions [31]. Instead a slight anisotropy was recorded for this structure with a factor 1.3 decrease in intensity between plasmonic modes recorded at 90 degrees shifted polarization positions. Figure 3d shows a SEM image of the analyzed trimer with an overlaid plot of the plasmon peak intensity variation in dependence of the polarization angle. The recorded anisotropy was attributed to slight imperfections in the symmetry of the studied trimer as close observation revealed that one particle was slightly elongated compared to the others.

Our results were in agreement with results recently reported by Tian et al. for silver nanoparticle assemblies where solvent evaporation-induced aggregation was used as process to create random and discrete nanoparticle assemblies which were subsequently analyzed by correlated electron microscopy/darkfield spectroscopy [32]. In spite of the high monodispersity of used nanocrystals the random nature of the assembly process lowered the degree of symmetry reached by formed structures. For this reason, Tian et al. found a mismatch between experimental and theoretical optical signatures of obtained trimers, of which geometry significantly deviated from the perfect $\mathrm{D} 3_{\mathrm{h}}$ symmetry. In our work, the yield of formed structures was significantly lower than what found by Tian et al. However, some examples of high symmetry clusters were found among formed structures. We argued that the formation of such clusters was facilitated by the use of a linker molecule with a bi-anchoring moiety and structural constraints (triple bond between Re atoms, short length and double bonds in the NCS group).

In order to further analyze dimer and trimer polarization behavior we calculated polarization resolved scattering spectra of the analyzed nanoparticle structures, shown in Figure 4.

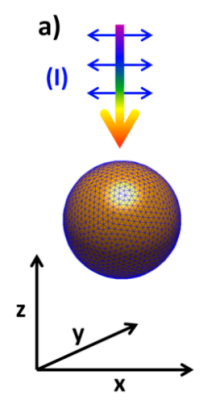

b)

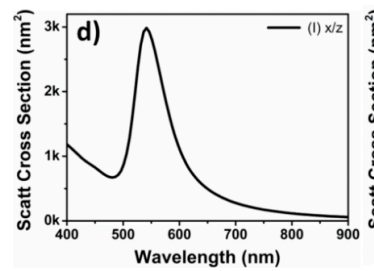

(III)
(I)

(II)
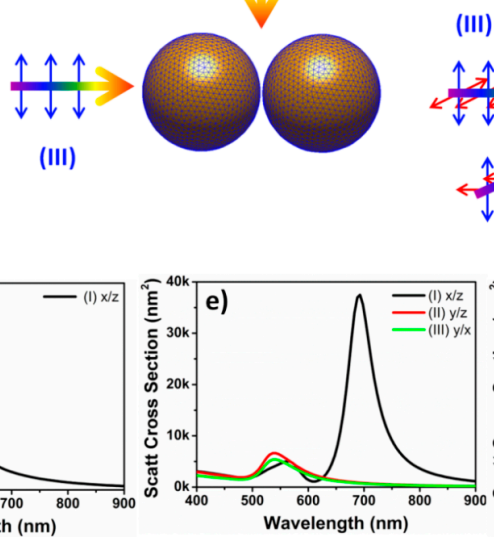

c)

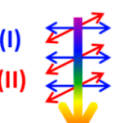

(III) (IV)

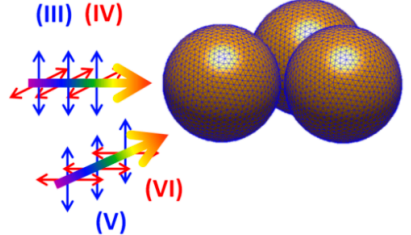

(V)

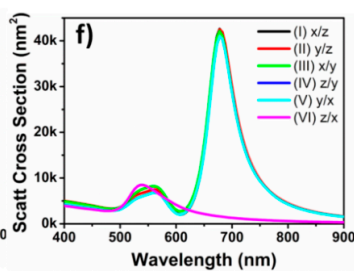

Figure 4. Schematic of a discretised $60 \mathrm{~nm}$ gold nanoparticle monomer (a), dimer (b) and trimer (c) with the considered incident light propagation directions (rainbow coloured arrow) and polarisation directions (blue and red double arrows) dependent on the nanostructure symmetry. Calculated scattering spectra for $60 \mathrm{~nm}$ gold nanoparticle monomers (d), dimers (e) and trimers (f) for the in panel $(\mathrm{a}-\mathrm{c})$ indicated incident light conditions (notation: pol/dir).

For perfectly symmetrical monomers only one light propagation and polarization direction (I) Figure $4 \mathrm{a}$ had to be considered and the calculated scattering spectrum showed one plasmon peak at $541 \mathrm{~nm}$. For a dimer with $\mathrm{D}_{\infty \mathrm{h}}$ symmetry three combinations of polarizations and propagation directions of incident light were considered: Light incident perpendicular to the dimer long axis with polarization direction parallel (I) and perpendicular (II) to the long axis of the dimer and 
light propagation direction parallel and light polarization perpendicular (III) to the dimer long axis. Additionally, due to the illumination geometry of the darkfield condenser a contribution of out-of-plane plasmon excitation was expected and the scattering spectrum was calculated for light incident parallel to the long dimer axis (III). At condition (I), the spectrum was dominated by a plasmon peak at $691 \mathrm{~nm}$, indicating strongly interacting dipoles arranged head to tail and excited with the electric field component parallel to the dimer long axis. In contrast, when the plasmon modes were excited perpendicular to the dimer long axis or out-of-plane, the spectra showed only one plasmon peak, centered at $539 \mathrm{~nm}$. This peak position was only slightly blue shifted compared to the plasmon peak of a single nanoparticle at $541 \mathrm{~nm}$ and indicated a very weak coupling of dipoles arranged shoulder to shoulder and excited with the electric field component perpendicular to the long axis of the dimer. The comparison between these calculated spectra and the experimentally recorded spectra of Figure 3 showed good agreement and indicated that the recorded spectra at any polarization angle are linear combinations of the calculated plasmon modes [5]. Moreover, our data are in agreement with theoretical data reported in literature showing that the plasmonic modes of a dimer are formed by hybridization of single particle modes and that, indeed, such structures can be treated as plasmonic molecules [33,34].

For the more complex structure of the nanoparticle trimer (Figure 4c) all possible incident light propagation and polarization directions were considered and the corresponding scattering spectra were calculated. For perpendicular incident light (I) and (II) identical scattering spectra were calculated with peaks at $678 \mathrm{~nm}$ and $562 \mathrm{~nm}$, indicating strong plasmon coupling irrespective of polarization direction. For incident light conditions (III) and (V) nearly identical scattering spectra to (I) and (II) were calculated, also originating from strong plasmon coupling. Only out-of-plane excitation (IV) and (VI) resulted in single peak scattering spectra $(539 \mathrm{~nm})$, showing very weak plasmon coupling. Again, as for the nanoparticle dimer, the calculated scattering spectra agreed well with the experimental data obtained for the trimer in Figure 3, which can be considered as linear combinations of the calculated plasmon modes. While for the nanoparticle dimer the two different plasmon modes can be understood as longitudinal and transversal plasmon modes, the case of a trimer is more complex. The origin of plasmon modes was analyzed by a model developed by Nordlander for the most symmetrical case of individual particles arranged in the form of an equilateral triangle [30]. According the group symmetry theory this plasmonic molecule trimer sustains nine dipolar plasmon modes. Six of the modes are in-plane, three can be classified as bonding and three are non-bonding, with energies lower and higher than that of an individual particle, respectively. Two bonding and two non-bonding modes are degenerate and can be excited by linearly polarized light so that the corresponding plasmon bands are located at a longer and shorter wavelength compared the individual particle, respectively. The state of lowest energy is dark for excitation with linearly polarized light, but can be excited with radially polarized light.

\section{Discussions}

Controlled addition of linker molecule led to formation of dimer, trimer and $n$-mer plasmonic structures, which were investigated by correlated electron microscopy/darkfield spectroscopy. Dimer structures were characterized by polarization-dependent scattering spectra and displayed maximum scattering intensity for polarized light excitation parallel to the long dimer axis. Trimer structures of $\mathrm{D}_{3 \mathrm{~h}}$ symmetry displayed slight polarization-dependent scattering spectra, in contrast to the lack of polarization theoretically expected from perfect equilateral triangle structures. The origin of scattering spectra in both dimer and trimer structures was elucidated by theoretical investigation. The experimental and theoretical studies confirmed that both dimer and trimer structures can be treated as plasmonic molecules and that their optical properties displayed characteristic predicted by the theoretical model of Nordlander developed for plasmonic dimers and trimers of $D_{3 h}$ symmetry. 


\section{Materials and Methods}

Materials: Citrate-stabilized gold nanocrystals with core diameter $d=60 \pm 6 \mathrm{~nm}$ were purchased from BBInternational (Cardiff, UK). Ultrapure deionized water with resistivity $\sim 18.2 \mathrm{M} \Omega \mathrm{cm}$ (ELGA PURELAB Ultra, Celbridge, Ireland) was used in all experiments. All glassware and cuvettes were cleaned using aqua regia and Piranha solutions prior use in order to prevent spurious aggregation of the nanocrystals. To improve the stability of the nanocrystals, $3 \mathrm{mM}$ aqueous tri-sodium citrate was added prior to use. Linker molecule $\operatorname{Re}_{2}(\mathrm{DMAA})_{4}(\mathrm{NCS})_{2}$ was synthesized according to literature [28] and was provided by Prof. Gallagher group at University College Cork.

Synthesis of gold nanocrystal clusters: The bi-functional linker molecule, $\operatorname{Re}_{2}(\mathrm{DMAA})_{4}(\mathrm{NCS})_{2}$ was dissolved in acetone (ACS grade). $3 \mathrm{~mL}$ of a 1:1 nanocrystal: tri-sodium citrate solution (1.5 $\mathrm{mL}$ of $1 \times 10^{-10} \mathrm{M}$ nanocrystal, mixed with $1.5 \mathrm{~mL}$ of $3 \mathrm{mM}$ aqueous tri-sodium citrate) was decanted into a clean cuvette. Aliquots of $10 \mu \mathrm{L} \mathrm{Re}_{2}(\mathrm{DMAA})_{4}(\mathrm{NCS})_{2}$ linker solution $(10 \mu \mathrm{M})$ were added at 10 minute intervals (typically four aliquots). The solution was mixed after each addition to ensure complete mixing of the nanocrystal/linker components.

Optical characterization: UV-Vis spectra of nanocrystal clusters were acquired with an Agilent/HP 8453 UV-Vis Spectrophotometer $(200 \mathrm{~nm}<\lambda<1100 \mathrm{~nm}$, Dublin, Ireland). The evolution of cluster formation was monitored by taking UV-vis spectra after each aliquot of linker addition.

Scattering spectra of individual nanocrystals and nanocrystal clusters were acquired with an inverted IX-71 Olympus microscope with an oil immersion darkfield condenser (Olympus U-DCW, 1.4 NA, Berlin, Germany) and 100 $\times$ objective (Olympus MPlanApo 100×/0.95 NA). Samples were illuminated with a $100 \mathrm{~W}$ halogen lamp. The light collected by the objective was directed either onto a color CMOS camera (DCC1645C, ThorLabs, Exeter, UK) for image acquisition or onto the entrance slit of a monochromator (SP-2356, Acton Research) equipped with a thermoelectrically cooled, back illuminated CCD (Spec10:100B, Princeton Instruments, Berlin, Germany) for spectra acquisition. The sample polarization rate was determined by a polarizer placed in collection between the sample and the monochromator. The experiments were performed by first recording an image of the sample with a mirror in the light path of the spectrometer. Once a suitable nanostructure was selected, the mirror was switched to a grating ( 300 groves $\mathrm{mm}^{-1}$ ) to disperse the scattered light and a spectrum was recorded using an acquisition time of $60 \mathrm{~s}$. Normalized scattering spectra from individual nanocrystals were obtained by subtracting and dividing by a background scattering spectrum taken from a nearby clean area on the sample.

Electron Microscopy: Scanning electron microscopy (SEM) images of gold clusters were acquired using a field emission SEM (JSM-6700F, JEOL UK Ltd., Welwyn Garden City, UK) operating at beam voltages of $10 \mathrm{kV}$. For navigation on the substrate, lithographically etched alignment marks in the Indium Tin Oxide (ITO) coating of glass cover slips were used.

Calculation of Nanostructure Scattering Spectra: To calculate the scattering spectra of gold nanoparticle monomers, dimers and trimers a boundary element method based toolbox for MATLAB was utilized.

\section{Conclusions}

In conclusion we have theoretically and experimentally investigated the optical properties of discrete plasmonic molecules formed by self-assembly of gold nanocrystals with bis-thioisocyanide linker molecules. The proposed rhenium bis-thioisocyanide complex linker constitutes an alternative to commonly used organic and biological linker molecules for formation of plasmonic molecules. Its compatibility with aqueous solutions provides a fast method for plasmonic structure production and its short length reduces the number of possible geometrical arrangements achievable for any data stoichiometry. Formed structures constitute promising building blocks for investigation of enhanced spectroscopies and to manipulate light at the nanoscale. 
Supplementary Materials: The following are available online at http://www.mdpi.com/2073-4352/6/9/117/s1, Figure S1: (a) $60 \mathrm{~nm}$ gold nanoparticle colloidal solution in front of a mixed background showing the difference between light scattered by and transmitted through the solution. (b) UV-Vis extinction spectrum of $60 \mathrm{~nm}$ gold nanoparticle colloidal solution. Figure S2: (a) Typical scattering image of an ensemble of gold nanocrystals; (b) schematic setup for darkfield imaging and spectra acquisition; (c) typical scattering spectrum of a single gold nanocrystal. Figure S3: $(a-b)$ Correlated darkfield scattering images-SEM images of various nanoparticle shapes found in a batch of $60 \mathrm{~nm}$ gold nanoparticle colloidal solution (scale bar $100 \mathrm{~nm}$; (c)) corresponding scattering spectra. Figure S4. SEM images of formed n-mer structures.

Acknowledgments: This work was supported by the European Commission under the FP7 NMP project "Funmol" (213382) and FP7 NMP project "Hysens" (263091).

Author Contributions: Carola Schopf conceived and designed experiments for Figures 2-4 and contributed to the writing of the manuscript. Ethel Noonan performed experiments for Figure 1 of the paper. Aidan J. Quinn conceived and designed experiments for Figure 1. Daniela Iacopino conceived experiments for Figures 2-4 and wrote the paper.

Conflicts of Interest: The authors declare no conflict of interest.

\section{References}

1. Brongersma, M.L.; Shalaev, V.M. The case for plasmonics. Science 2010, 328, 440-441. [CrossRef] [PubMed]

2. Zhang, J.G.; Noguez, C. Plasmonic optical properties and applications of metal nanostructures. Plasmonics 2008, 3, 127-150. [CrossRef]

3. Pescaglini, A.; O'Riordan, A.; Quinn, J.A.; Iacopino, D. Controlled assembly of Au nanorods into 1D architectures by electric field assisted deposition. J. Mater. Chem. C 2014, 2, 6810-6816. [CrossRef]

4. Schopf, C.; Martín, A.; Burke, M.; Jones, D.; Pescaglini, A.; O’Riordan, A.; Quinn, A.J.; Iacopino, D. Au nanorod plasmonic superstructures obtained by a combined droplet evaporation and stamping method. J. Mater. Chem. C 2014, 2, 3536-3541. [CrossRef]

5. Martín, A.; Schopf, C.; Pescaglini, A.; Wang, J.J.; Iacopino, D. Facile formation of ordered vertical arrays by droplet evaporation of Au nanorod organic solutions. Langmuir 2014, 30, 10206-10212. [CrossRef] [PubMed]

6. Zheng, J.; Birktoft, J.J.; Chen, Y.; Wang, T.; Sha, R.; Constantinou, P.E.; Ginell, S.L.; Mao, C.; Seeman, N.C. Nature 2009, 461, 74-77. [PubMed]

7. Zeng, X.; Liu, F.; Fowler, A.G; Ungar, G.; Cseh, L.; Mehl, H.G.; Macdonald, J.E. 3D ordered gold strings by coating nanoparticles with mesogens. Adv. Mater. 2009, 21, 1746-1750. [CrossRef]

8. Wustholz, K.L.; Henry, A.; McMahon, J.M.; Freeman, M.R.; Valley, N.; Piotti, M.E.; Natan, M.J.; Schatz, G.C.; Van Duyne, R.P. Structure-activity relationships in gold nanoparticle dimers and trimers for surface-enhanced Raman spectroscopy. J Am. Chem. Soc. 2010, 132, 10903-10910. [CrossRef] [PubMed]

9. Lim, D.K.; Jeon, K.S.; Kim, H.M.; Nam, J.M.; Suh, Y.D. Nanogap-engineerable Raman-active nanodumbbells for single-molecule detection. Nat. Mater. 2010, 9, 60-67. [CrossRef] [PubMed]

10. Fu, Y.; Zhang, J.; Lakowicz, J.R. Plasmon-Enhanced Fluorescence from Single Fluorophores End-Linked to Gold Nanorods. J. Am. Chem. Soc. 2010, 132, 5540-5541. [CrossRef] [PubMed]

11. Maier, S.A.; Kik, P.G.; Atwater, H.A. Observation of coupled plasmon-polariton modes in Au nanoparticle chain waveguides of different lengths: Estimation of waveguide loss. Appl. Phys. Lett. 2002, 81, 1714-1716. [CrossRef]

12. Reinhard, B.M.; Sheikholeslami, S.; Mastroianni, A.; Aliviatos, A.P.; Liphard, J. Use of plasmon coupling to reveal the dynamics of DNA bending and cleavage by single EcoRV restriction enzymes. Proc. Natl. Acad. Sci. USA 2007, 104, 2667-2672. [CrossRef] [PubMed]

13. Endo, T.; Kerman, K.; Nagatani, N.; Hiepa, H.M.; Kim, D.K.; Yonezawa, Y. Multiple label-free detection of antigen-antibody reaction using localized surface plasmon resonance-based core-shell structured nanoparticle layer nanochip. Anal. Chem. 2006, 78, 6465-6475. [CrossRef] [PubMed]

14. Alu, A.; Engheta, N. The quest for magnetic plasmons at optical frequencies. Opt. Express 2009, 17, 5723-5730. [CrossRef] [PubMed]

15. Alu, A.; Salandrino, A.; Engheta, N. Negative effective permeability and left-handed materials at optical frequencies. Opt. Express 2006, 14, 1557-1567. [CrossRef] [PubMed]

16. Prodan, E.; Nordlander, P. Plasmon hybridization in spherical nanoparticles. Chem. Phys. 2004, 120, 5444-5454. [CrossRef] [PubMed] 
17. Sethi, M.; Joung, G.; Knecht, M.R. Linear assembly of Au nanorods using biomimetic ligands. Langmuir 2009, 25, 1572-1581. [CrossRef] [PubMed]

18. Matsuda, K.; Yamaguchi, H.; Sakano, T.; Ikeda, M.; Tanifuji, N.; Irie, M. Conductance Photoswitching of Diarylethene-Gold Nanoparticle Network Induced by Photochromic Reaction. J. Phys. Chem. C 2008, 112, 17005-17010. [CrossRef]

19. Caswell, K.K.; Wilson, J.N.; Bunz, U.H.F.; Murphy, C.J. Preferential end-to-end assembly of gold nanorods by biotin-streptavidin connectors. J. Am. Chem. Soc. 2003, 125, 13914-13915. [CrossRef] [PubMed]

20. Ongaro, A.; Griffin, F.; Beecher, P.; Nagle, L.; Iacopino, D.; Quinn, A.; Redmond, G.; Fitzmaurice, D. DNA-Templated Assembly of Conducting Gold Nanowires between Gold Electrodes on a Silicon Oxide Substrate. Chem. Mater. 2005, 17, 1959-1964. [CrossRef]

21. Eritja, R.; Avino, A.; De la Torre, B.; Fitzmaurice, D.; Ongaro, A.; Stanca, S.E.; DiSalvo, A.; Manning, B.; Iacopino, D. A Flexible Method for the Fabrication of Gold Nanostructures Using Oligonucleotide Derivatives. Nucleos. Nucleot. Nucl. 2007, 26, 1605-1609. [CrossRef] [PubMed]

22. Zheng, J.W.; Constantinou, P.E.; Micheel, C.; Alivisatos, P.A.; Kiehl, R.A.; Seeman, N.C. Two-Dimensional Nanoparticle Arrays Show the Organizational Power of Robust DNA Motifs. Nano Lett. 2006, 6, 1502-1504. [CrossRef] [PubMed]

23. Qian, X.; Emory, S.R.; Nie, S. Anchoring Molecular Chromophores to Colloidal Gold Nanocrystals: Surface-Enhanced Raman Evidence for Strong Electronic Coupling and Irreversible Structural Locking. J. Am. Chem. Soc. 2012, 134, 2000-2003. [CrossRef] [PubMed]

24. Thompson, D.; Liao, J.; Nolan, M.; Quinn, A.J.; Nijhuis, C.A.; O’Dwyer, C.; Nirmalraj, P.N.; Schönenberger, C.; Michel, C. Formation Mechanism of Metal-Molecule-Metal Junctions: Molecule-Assisted Migration on Metal Defects. J. Phys. Chem. C 2015, 119, 19438-19451. [CrossRef]

25. Chen, J.; Huang, Y.; Zhao, S.; Lu, X.; Tian, J. Gold nanoparticles-based fluorescence resonance energy transfer for competitive immunoassay of biomolecules. Analyst 2012, 137, 5885-5890. [CrossRef] [PubMed]

26. Shi, X.; Wang, S.; Meshinchi, S.; Van Antwerp, M.E.; Bi, X.; Lee, I.; Baker, J.R., Jr. Dendrimer-Entrapped Gold Nanoparticles as a Platform for Cancer-Cell Targeting and Imaging. Small 2007, 3, 1245-1252. [CrossRef] [PubMed]

27. Sikora, M.; Szymczak, P.; Thompson, D.; Cieplak, M. Linker-mediated assembly of gold nanoparticles into multimeric motifs. Nanotechnology 2011, 22, 445601. [CrossRef] [PubMed]

28. Donocadh, L.P.; Spalding, T.; Gallagher, J. Substitution reactions of rhenium-chloride bonds in [Re2(DMAA)4C12], (DMAA = 2',6'-dimethylacetanilido); synthesis and characterisation of [Re2(DMAA)4X2] $(\mathrm{X}=\mathrm{NCO}, \mathrm{NCS}, \mathrm{N} 3)$ and $[\operatorname{Re} 2(\mathrm{DMAA}) 4 \mathrm{Ln}][\mathrm{SbF} 6] 2\left(\mathrm{n}=2, \mathrm{~L}=\right.$ pyridine; $\mathrm{n}=1, \mathrm{~L}=4,4^{\prime}$-bipyridine). Polyhedron 2003, 22, 1281-1287.

29. Hohester, U.; Trügler, A. MNPBEM-A Matlab toolbox for the simulation of plasmonic nanoparticles. Comput. Phys. Commun. 2012, 183, 370-371. [CrossRef]

30. Johnson, P.B.; Christy, R.W. Optical Constants of the Noble Metals. Phys. Rev. B 1972, 6, 4370-4380. [CrossRef]

31. Brandl, D.W.; Mirin, N.A.; Nordlander, P. Plasmon Modes of Nanosphere Trimers and Quadrumers. J. Phys. Chem. B 2006, 110, 12302-12310. [CrossRef] [PubMed]

32. Tian, X.; Zhou, Y.; Thota, S.; Zou, S.; Zhao, J. Plasmonic Coupling in Single Silver Nanosphere Assemblies by Polarization-Dependent Dark-Field Scattering Spectroscopy. J. Phys. Chem. C 2014, 118, 13801-13808. [CrossRef]

33. Zohar, R.N.; Chuntonov, L.; Haran, G. The simplest plasmonic molecules: Metal nanoparticle dimers and trimmers. J. Photochem. Photobiol. C Photochem. 2014, 21, 26-39. [CrossRef]

34. Nordlander, P.; Oubre, C.; Prodan, E.; Li, K.; Stockman, M. Plasmon Hybridization in Nanoparticle Dimers. Nano Lett. 2004, 4, 899-903. [CrossRef]

(C) 2016 by the authors; licensee MDPI, Basel, Switzerland. This article is an open access article distributed under the terms and conditions of the Creative Commons Attribution (CC-BY) license (http://creativecommons.org/licenses/by/4.0/). 